\title{
A CHEAP FORM OF SELF-REGULATING GAS GENERATOR.
}

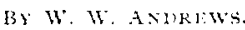

Ki.

THIS form of generator is so cheap and easily set up that it makes it possible for every teacher and experimenter in chemistry to have, at practically no expense, a set of generators
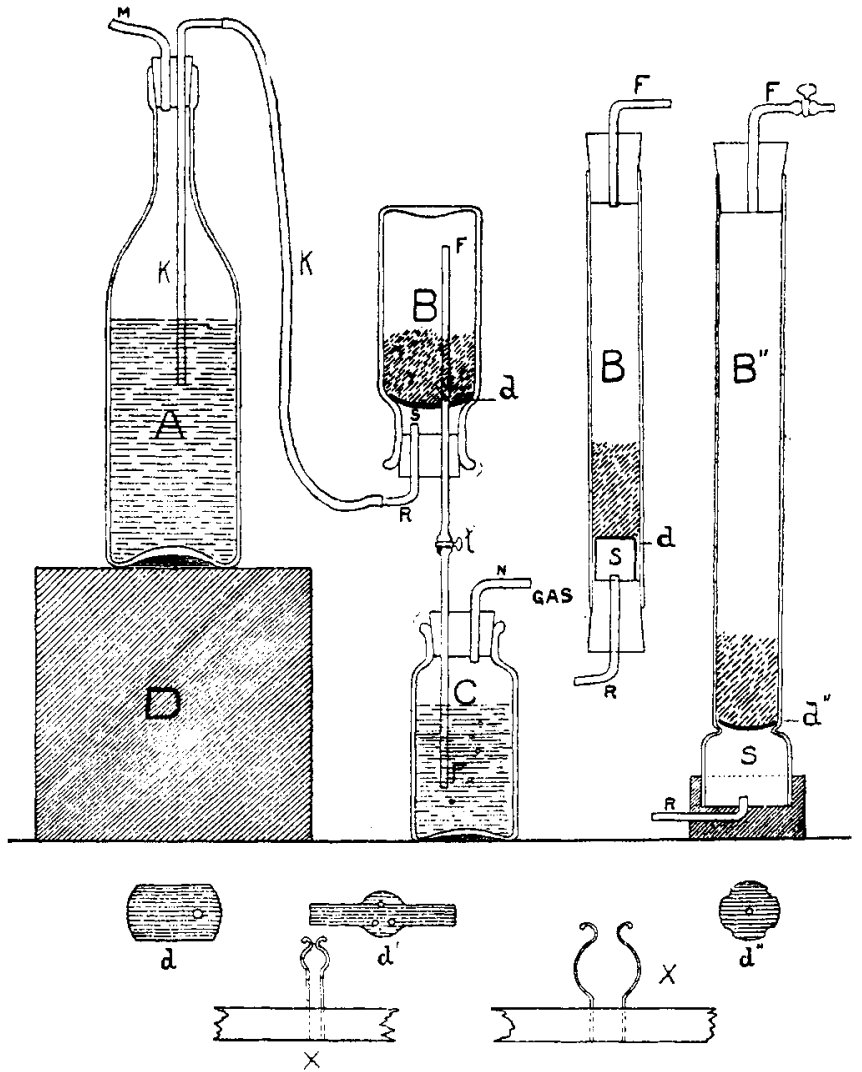

capable of yielding, whenever called upon, a supply of hydrogen, hydrogen sulphide, chlorine, carbon dioxide, sulphur dioxide, etc. It consists of an ordinary bottle, $\mathrm{A}$, to serve as reservoir 
for the acid, a smaller bottle, B, which is the generator proper, and $\mathrm{C}$, the familiar wash-bottle. $\mathrm{A}$ is closed by a cork holding two pieces of glass tubing. The one in just pierces the cork and the other, $\mathrm{K}$, reaches down a short distance below the surface of the acid and is connected by means of a rubber tube with $R$, thus forming a siphon leading from $A$ to $B$, as shown in cut. The tube $M$ is used to start the siphon $K R$ by blowing into $A$ while the stop-cock, $t$ is open. $\mathrm{F}$ is the exit tube for the gas. The charge of solid in B rests on a shelf of sheet lead, $d$, the width of which equals the diameter of the neck of the bottle, and its length the internal diameter of the bottle. It serves the purpose of securing a space, $\mathrm{s}$, which prevents the extra gas generated, when $t$ is closed, from pressing the acid out of the siphon. Instead of a bottle for holding the charge a calcium chloride tube, or an Argand lamp chimney, with its larger end closed with a cork or sunk into a wooden block and cemented with paraffin wax, or even a piece of large-sized tubing may be used. If the last be used the space $\mathrm{s}$ is secured by cutting the lead in the shape $d^{\prime}$ and bending it to form a bench to support the solid charge, as shown. $d^{\prime \prime}$ is the shape of the lead support to be used in the calcium chloride tube or Argand chimney. $\mathrm{x}$ and $\mathrm{x}^{\prime}$, are the wire springs for holding B to its support, from which it may be lifted or replaced instantly. One is to enclasp the body and the other the neck of the bottle, or one of the glass tubes below the cork.

The advantages of this form of generator are:

I. Its cheapness. An ordinary fruit-juice bottle and two widemouthed eight-ounce bottles will, with the necessary tubing, make one of good capacity.

2. Its convenience and safety. It is strictly self-regulating. If a rubber tube fitted with a glass plug one-half inch long, instead of a stop-cock be used, the gas flows only when the fingers pinch up the rubber along one side of the plug and ceases the moment they are lifted. The apparatus, therefore, is self-closing. It is very easy to recharge either with acid or solid.

3. It ensures a more even quality of gas than the well-known $K$ ipp, and as complete utilization of the acid as the Koninck generator. When the gas presses the acid back from $B$, it enters $A$ laden with dissolved solid and, on account of its greater specific gravity, 
it falls in a straight line to the bottom, where it spreads out in a dense layer. When the apparatus is again set working, the purest acid is drawn from the npper layers. There is, consequently, some advantage in using a tall bottle for $\mathrm{A}$.

4. It may be put together in a form which has all the advantages possessed by the Schanche generator (see this JoukNAL, Dec. 1894 .) Making the glass tube $\mathrm{R}$, long enough to reach to the top of $B$, and to curve a little downward, so that the acid may drop on the top of the solid charge and trickle through it. A drainage-tube with stop-cock must be inserted into the cork to carry off the used-up acid from the space 5 . The inverted bottle for $B$, possesses this advantage over the calcium chloride tube or chimney; viz., that owing to the position of the mouth of the gas exit tube $F$, solid particles are not so likely to be shot into it when the acid is vigorously attacking the solid charge.

5. The pressure can be regulated at will by placing $A$ at different heights, and this pressure may, at any monent, be reinforced by blowing into $A$, and the reinforcement held by closing $M$ by means of a rubber tube and pinch-cock.

\section{SOME OF THE PROPERTIES OF CALCIUM CARBIDE.}

BY F. P. VENABLE ANU THOMAS CLAKKE

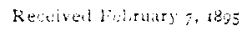

THE calcium carbide used was prepared by the Willson Aluminum Company. In this preparation, lime is mixed with some form of carbon, as coal-tar; the mass is then heated, with stirring, until a thorough mixture is obtained. The proportions are so arranged that the mass becones dry and hard on cooling. This mass, in lumps, is then placed in the electric furnace. In a very short time after the turning on of the current, the process is complete. The molten mass can be run out of the crucible or it may be removed after cooling. On examination, it is easy to see that there is more or less of carbon unchanged, or rather converted into the graphitic variety by the intense heat. Along with this are to be seen crystalline masses, lustrous and dark brownish black it color.

These are quite hard, and break with a crystalline fracture. Several efforts at effecting a separation from the graphitic carbon 